$\xi=-1$ 圆

\title{
The effect of locally source carbonaceous materials on the mechanical properties of mild steel
}

\author{
Dongo, E. I ${ }^{1 *}$, Seidu, S. $\mathbf{O}^{2}$, Ogbodo, F .J ${ }^{1}$ \\ ${ }^{1}$ Department of Metallurgical Engineering, Kogi State Polytechnic, Lokoja \\ ${ }^{2}$ Department of Metallurgical and Materials Engineering, Federal University of Technology, Akure \\ *Corresponding author E-mail: isaacdongo@gmail.com
}

\begin{abstract}
The work focuses on the effects of locally sourced carbonaceous materials such as Animal bone, Snail shell and Periwinkle Shell on the mechanical properties of mild steel carburized at varying treatment temperatures of $700,800,900,1000$ and $1100{ }^{\circ} \mathrm{C}$, soaked at varying carburizing time of $5 \mathrm{hr}, 4 \mathrm{hr}, 3 \mathrm{hr}, 2 \mathrm{hr}$ and $1 \mathrm{hr}$ at the respective treatment temperatures. The samples are quenched in oil and tempered at $350^{\circ} \mathrm{C}$ for 45 minutes. Prior to the carburization process, standard test samples were prepared from the as received specimen for tensile tests, wear test and micro hardness analysis. After the carburization process, the test samples were subjected to standard test to generate data for ultimate tensile strength, the case and core hardness values, wear rate and wear resistance of the carburized samples. The study shows that the mechanical properties of mild steels were found to be strongly influence by each of the carbonaceous materials.
\end{abstract}

Keywords: Animal Bone; Hardness; Pack Carburization; Periwinkle Shell; Snail Shell.

\section{Introduction}

Low carbon steel being the greatest quantity of steel produced generally contains less than about $0.25 \%$ of carbon [2]. By reasons of its dominance and workability among the classes of steel, it has found a wide application in the production of engineering parts and applications like gears, cams, and crankshafts where high hardness on the outer surface and softer core is desire and tough enough to withstand shocks. To attain such properties it is very difficult to employ a low carbon steels (because it cannot be harden) or high carbon steels (because of its poor toughness). In order to obtain the required properties, a medium carbon steels is required as it combines both the properties of the above-mentioned steels.

Literatures in recent times has revealed that most of these hightech equipment and facilities for carburizing treatment of Low carbon steel with which fabricators and mechanist easily use to produce their parts naturally lacks the required mechanical properties [4]. However, carburization is one of the most commonly performed steel heat treatments performed by packing the low carbon wrought iron parts in a carbonaceous material, then Raising the temperature of the pack to a predetermined temperature and holding (soaking) for some specified time and allow cooling down using a quenching media. The surface of the parts becomes very hard while the core is very soft which retained the toughness of the low carbon steel [7].

However, due to the increasing demand for material that necessitate hard wear resistance in order to withstand some properties such as cutting, abrasion, heat resistance, warping etc the work focus on a low carbon steel material to obtain a structure having a hard surface with soft core which is capable of withstanding dynamic stress. The study is specifically to unearth the possibility of using some local carbonaceous material such as animal bone, snail shell and periwinkle shells to improve certain mechanical proper- ties via carburization processes. It is intended to also determine the case depth and to compare their impact on the surface treatment on each sample. Higgins [8] describes Carburization as a diffusion-controlled process, in which the longer the steel is held in the carbon environment the greater the carbon penetration will be and the higher the carbon content.

\section{Experimental methodology}

Specimen samples of low carbon steel of known chemical composition (Table 1) is sourced from the Universal Steel Lagos and are cut into a specification compatible for Micro Hardness Test, Tensile Testing machine, Wear Resistance Test, and Micrographic Examination. The carbonaceous materials under investigation are the Animal Bone (A), Periwinkle Shell (P), Snail Shell (S) prepared in granular form is mixed with $20 \%$ lime $\left(\mathrm{CaCO}_{3}\right)$ which serves as the energizer, and the homogenized mixtures are then packed separately with the required number of the samples in the three carburized boxes fabricated from mild steel.

\begin{tabular}{llllllllllll}
\multicolumn{10}{c}{ Table 1: Chemical Composition of Mild Steel Rod } \\
\hline $\begin{array}{l}\text { Ele- } \\
\text { ments }\end{array}$ & $\mathrm{C}$ & $\mathrm{Si}$ & $\mathrm{Mn}$ & $\mathrm{S}$ & $\mathrm{P}$ & $\mathrm{Cr}$ & $\begin{array}{l}\mathrm{Cu} \\
\# 1\end{array}$ & $\begin{array}{l}\mathrm{Al} \\
\# 1\end{array}$ & $\mathrm{Ti}$ & $\mathrm{Fe}$ \\
\hline $\begin{array}{l}\text { Aver- } \\
\text { age }\end{array}$ & 0.1 & 0.1 & 0.6 & 0.0 & 0.0 & 0.1 & 0.2 & 0.0 & 0.0 & 98. \\
$\begin{array}{l}\text { Com- } \\
\text { posi- } \\
\text { tion }\end{array}$ & 0 & 45 & 18 & 05 & 41 & 04 & 64 & 43 & 05 & 452 \\
& 0 & 0 & 0 & 0 & 0 & 0 & 0 & 0 & 0 \\
\hline
\end{tabular}

Source: Universal Steel Lagos

Each identified carburizing boxes were packed with specified number of the work samples covered with their respective carburizing materials and sealed with clay to prevent the escape of the carbonaceous material. it is then charged into the furnace (Muffle Furnace) having a maximum temperature capacity of $1200 \mathrm{C}$ to 
austenitize the carburized steel samples at various heat treatment temperature ranging from $700^{\circ} \mathrm{C}(\mathrm{T} 1), 800^{\circ} \mathrm{C}(\mathrm{T} 2), 900^{\circ} \mathrm{C}(\mathrm{T} 3)$, $1000^{\circ} \mathrm{C}(\mathrm{T} 4), 1100^{\circ} \mathrm{C}(\mathrm{T} 5)$ at a soaking time of $5 \mathrm{hrs}$, $4 \mathrm{hrs}$, $3 \mathrm{hrs}$, $2 \mathrm{hrs}, 1 \mathrm{hr}$ respectively to allow for carbon diffusion. Three different metal containers of sufficient volumetric capacity were adapted as baths for quenching purpose using industrial oil as the quenchant. The materials were there after tempered at $350^{\circ} \mathrm{C}$ with a soaking time of $45 \mathrm{~min}$ and then allowed to cool in free air for all the samples. The samples were identified by their respective carburizing medium and heat-treated temperatures i.e at treatment temperature of $700^{\circ} \mathrm{C}$ snail is identified as ST1, while PT1 is periwinkle and AT1 is identified as the animal bone. Similarly, other treatment temperatures are identified in this order.
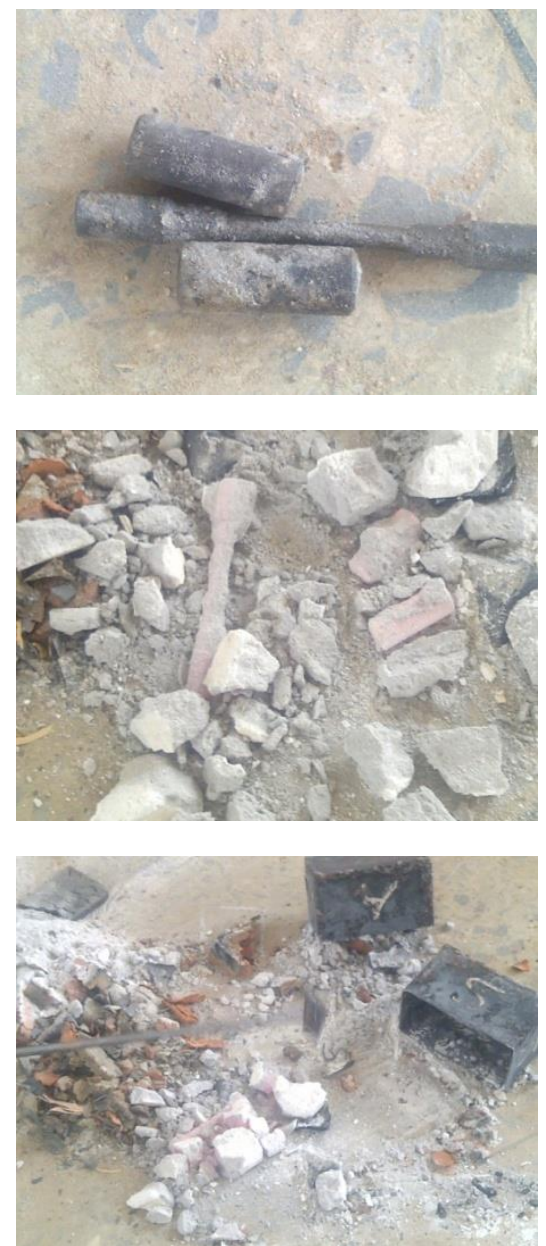

\section{Mechanical property test}

\subsection{Tensile testing}

The entire tensile test specimen was subjected to tensile loads one after the other until the material fails or fracture using the Instron universal testing machine model number 3360 at the Engineering
Materials Development Institute (EMDI) Akure. The machine automatically generates data and graph.

\subsection{Micro-hardness testing}

The sample was prepared through the cross-section (grinded and polish) after which they were mounted on the Micro- Hardness Testing machine for hardness measurements. The distances of every impression on the surface samples is determined from the measuring device on the machine where five hardness measurements were taken (at a regular interval of $1 \mathrm{~mm}$ from the case to the core) per specimen and the average depth of each sample was determined. Readings of the control sample was also taken and then compared with the readings from the samples.

\subsection{Wear rate}

Wear rates are calculated results reflecting wear mass loss, volume loss or linear dimension change under unit applied normal force or unit sliding distance. The Wear test was carried out for two specific reasons:

1) From a material point of view, the test is performed to evaluate the wear property of a material to determine whether the material is adequate for a specific wear application.

2) From a surface engineering point of view, the wear test is carried out to evaluate the potential of using a certain surface engineering technology to reduce wear for a specific application, and to investigate the effect of treatment conditions (processing parameters) on the wear performance, so that optimized surface treatment conditions can be realized.

\subsection{Wear resistance}

Wear resistance is a term frequently used to describe the anti-wear properties of a material. However, the scientific meaning of wear resistance is vague, and there is no specific unit to describe wear resistance. Nevertheless, the inverse of mass loss or volume loss was used as the (relative) wear resistance. The ratio of wear loss for a reference material over that of the investigated material under same testing conditions was adopted as the relative wear resistance.

The surface of the sample to be tested was made parallel to the surface of the rotating disc of the machine. Care was taken to ensure that sample under test is firmly held to the surface of the rotating disc during the test so that the sample does not fling out while running the test.

\section{Results and discussion}

Summary of the analyzed data and its results are presented with relevant interpretations in tables and figures

\subsection{Tensile strength test}




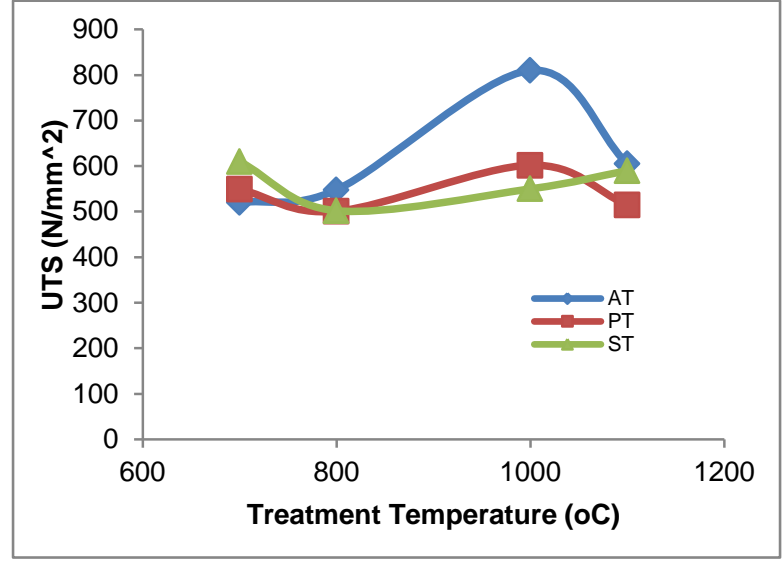

Fig. 1a: Variation of strength (UTS).

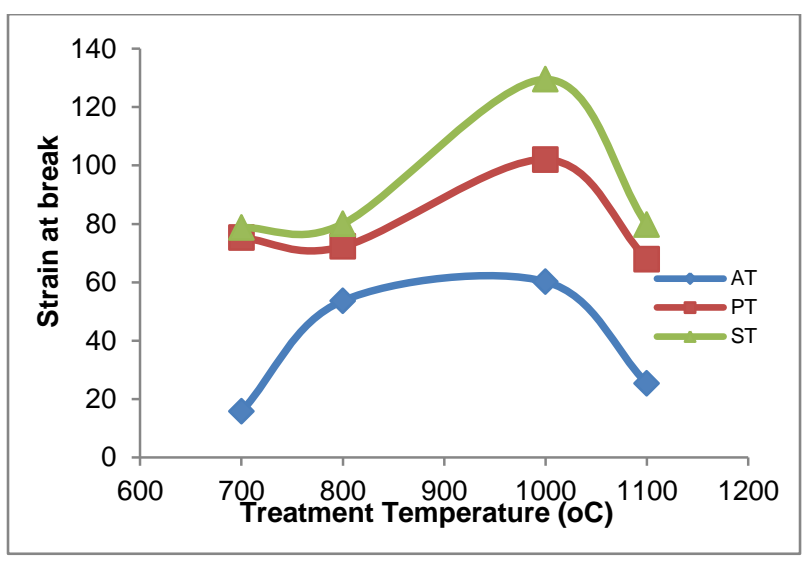

Fig. 1b: Variation of Tensile Strain at Break.

The result shows that animal bone (AT) has the highest UTS value followed by periwinkle shell (PT) and snail shell (ST) respectively. This is an indication of high rate of carbon diffusion deposited on the samples. Hence, the higher the UTS the less ductile the material becomes, this is in agreement with the general rule that the more ductile a material is, the less are its tensile and yield strength (fig1a). However, the values of strain to fracture of the three carburizing materials at varying temperature shows that they all exhibits high strain to fracture at $1000^{\circ} \mathrm{C}$ with snail having the highest strain value (129.4872) among them, followed by Periwinkle (102.0521) and animal bone been the least (60.21015) as evident in fig $1 \mathrm{~b}$.

Summarily from above charts, the study shows that Animal bones at $1000^{\circ} \mathrm{C}$ (AT4) fig 1a posses the highest ultimate tensile stress $\left(810 \mathrm{~N} / \mathrm{mm}^{2}\right)$ and tensile stress at Break fig $1 \mathrm{~b}(800.8628 \mathrm{~mm})$, this is because the presence of martensite structure in quenched steel greatly increases hardness and tensile strength [6]. The matensitic structure is certainly due to the hardenability, which indicates high deposition of carbon on the material. This also explains the facts that the rate of diffusion of carbon into the steel depends on the carburizing temperature, the composition of the carburizing mix- ture and the type of steel [1]. The carbon content in the case becomes higher as temperatures are increased this is because both temperature and time plays important factors in carburizing.

\subsection{Abrasion wear test}

Figures $2(\mathrm{a} \& \mathrm{~b})$ shows the wear properties of the developed microstructure after casehardening; from the result, it could be deduced that an inverse relationship was established between the resistance and rate of the material under the prevailing condition. Observation shows that sample AT2 casehardened with animal bone $800^{\circ} \mathrm{C}$ has the highest wear resistance as it possess the highest resistance value of $9.819 \times 10^{7} \mathrm{~cm}^{2}$, thus exhibiting the lowest wear rate when compared with other carbonaceous material. The reason for this explicit result could be attributed to its rate of diffusivity into the material via the surface/case.

\subsection{Micro hardness test results}

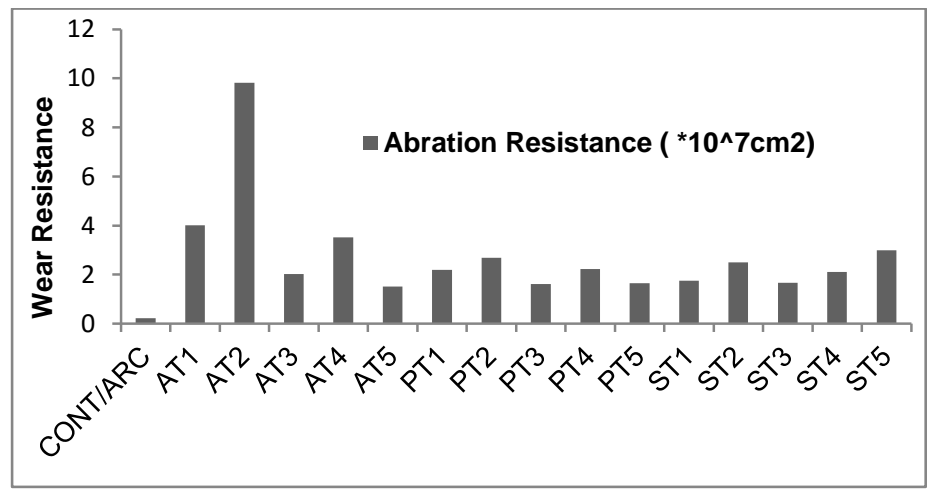

Fig. 2a: Variation of Wear Resistance. 


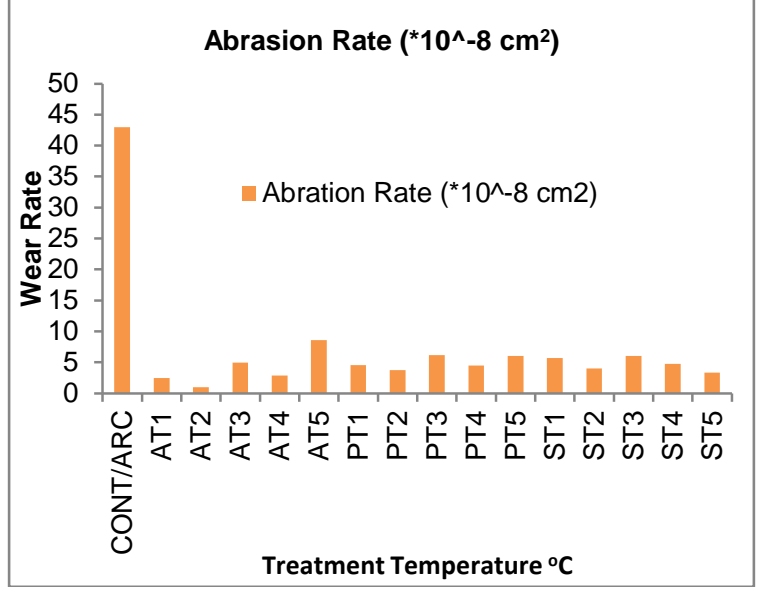

Fig. 2b: Variation of Wear Rate.

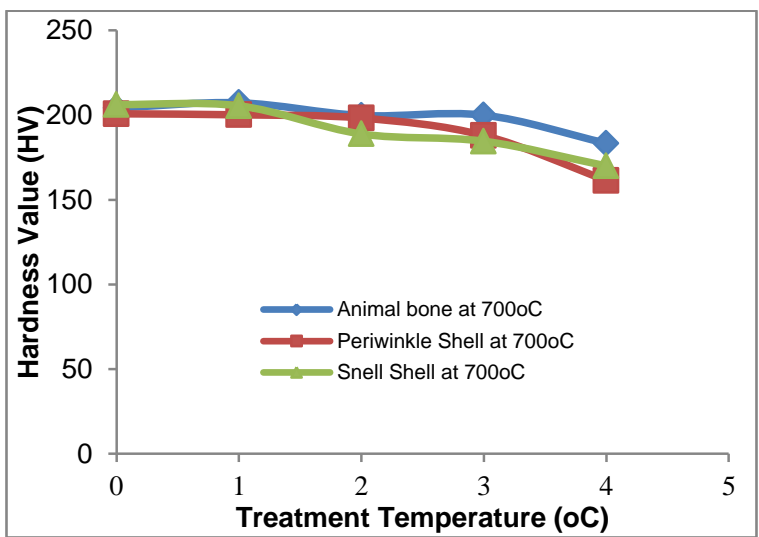

Fig. 3: Variation of Hardness Values at 700oc.

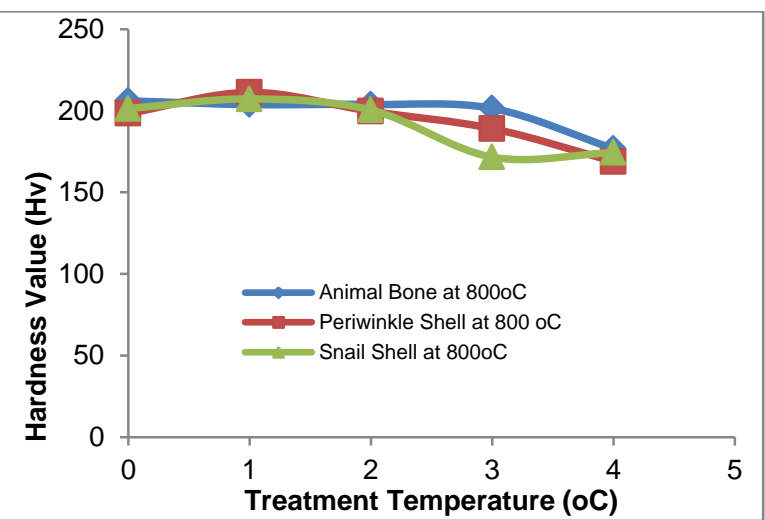

Fig. 4: Variation of Hardness Values at $800 \mathrm{oC}$.

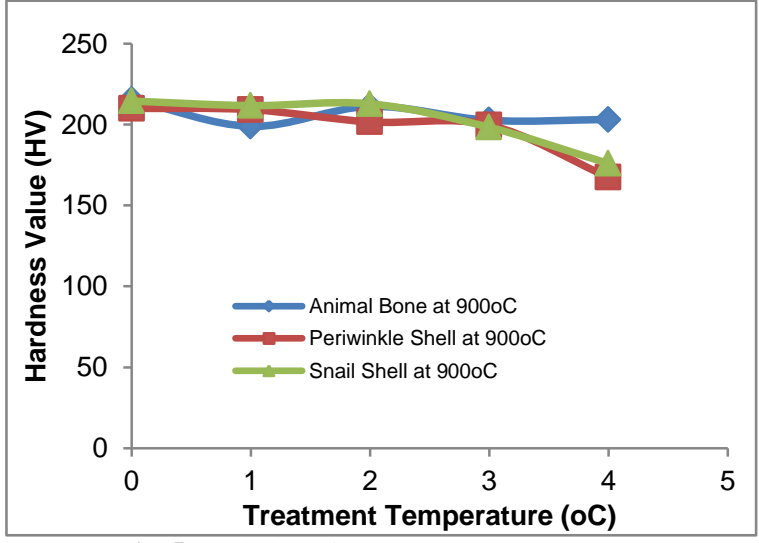

Fig. 5: Variation of Hardness Values at 900oC. 


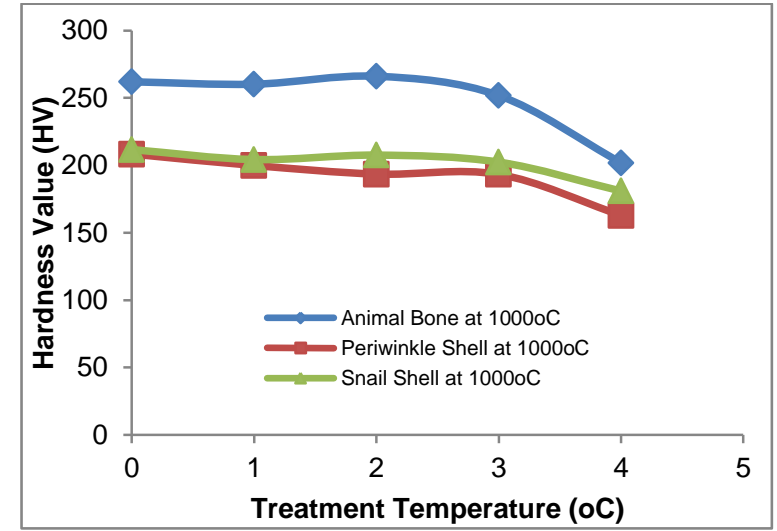

Fig. 6: Variation of Hardness Values at 1000oC.

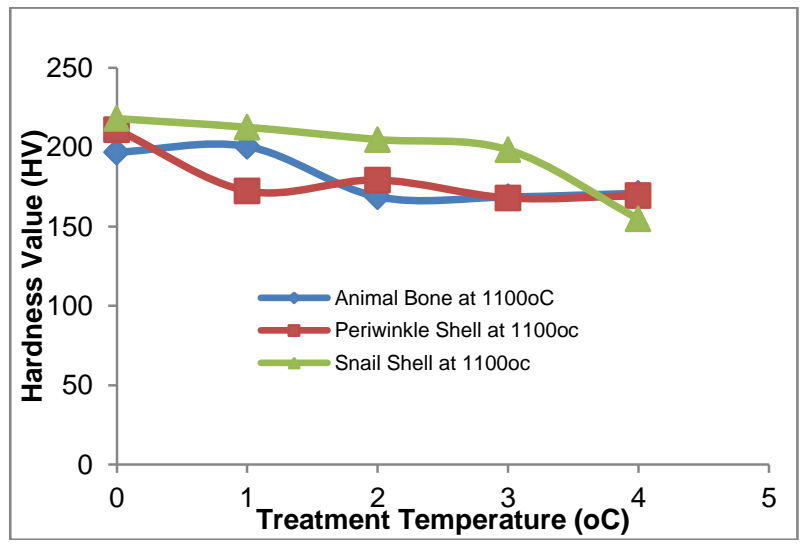

Fig. 7: Variation of Hardness Values at 1100oC.

The amount of carbon present in plain carbon steel has a pronounced effect on the properties of steel and on the selection of suitable heat treatments to attain certain desired properties. In order to assess the effect of the selected carbonaceous material in the hardness property of the material, micro-hardness test was conducted from the case to the core (0-5) at a regular interval of $1 \mathrm{~mm}$. the results are shown in Figure 3- 7, a particular parabolic trend was observed in all the profiles. An initial tremendous increase in the hardness was observed showing that the carbonaceous material selected has a direct effect on most of the case of the material with the 0 's and 1 possessing the maximum hardness values. This high value was however observed to gradually reduce with respect to distance toward the core of the samples. This arises because of the diffusion of high carbide content at the surface in which the effects at the core remains chemically unaffected or with negligible effect. When heat-treated, the high-carbon surface responds to hardening, and the core toughens.

Summarily, the study reveals that animal bone diffuses the highest percentage of carbon on the specimen at $1000^{\circ} \mathrm{C}$ (AT4), while snail shell diffuses its highest carbon at $1100^{\circ} \mathrm{C}$ (ST5). This explains the fact that carbon diffuses into steel at high temperatures for specified time (few hours to a day) and the carbon gradient produced decreases from the surface into the steel [5].

\section{Conclusion}

- From the various results obtained, it can be deduce that animal bone, periwinkle shell and snail shell are suitable carbonaceous materials at varying elevated temperatures.

- The micro hardness value depicts that the carbon deposited on the surface of the mild steel diffuses into the steel through the case to the core, which implies that case depth can be increased by longer cycle of carburization and exponentially by increasing the carburization temperature. Result indicates that snail shell with respect to increase in temperature has consistency in its hardness value.
- $\quad$ The samples having greater case depth and surface hardness has more wear resistant than that with low case depth and low surface hardness. Thus, the study has revealed that animal bone; snail shell and periwinkle shell in that order show evidence of good wear resistance and hardness value, which makes them suitable for increasing surface hardness and improves wear resistance for mild steel.

- The tensile strength of the samples carburized in animal bone at $1000^{\circ} \mathrm{C}$ shows higher value than periwinkle and snail shell. The Micrographs indicated that the surfaces of the heat-treated samples are martensitic which also correlates with the micro hardness values showing the rate of carbon deposition from the case to the core.

\section{Acknowledgment}

The authors wish to appreciate Engr. Momoh Monday Itopa of The Department of Metallurgical Engineering, School of Engineering, Kogi State Polytechnic, Lokoja Nigeria. His contribution in the area of data collection is highly appreciated. We acknowledge TETFund for the sponsorship.

\section{References}

[1] A. Oyetunji and S.O Adeosun, Effects of Carburizing Process Variables on Mechanical and Chemical Properties of Carburized Mild Steel, Journal of Basic \& Applied Sciences, 8, 319, 2012. http://dx.doi.org/10.6000/1927-5129.2012.08.02.11.

[2] D. W Callister Jr, Material Science and Engineering an Introduction, John Wiley and son Inc, New York: USA, 1987.

[3] H. C Child, Surface hardening of steel, University Press, Pp10-30, $1979 \mathrm{Http} / /$; en; Wikipedia. Org/Carbon Steel .htm.

[4] J. E Ohize, Effects of Local Carbonaceous Materials on the Mechanical Properties of Mild Steel, 2009.

[5] J. I Goldstein and E. A Moren, Diffusion Modeling of the carburization Process, American Society for Metals and the Metallurgical Society of AIME. vol. 9A, 1978. 
[6] P. A Ihom, G.B Nyior,J I Nor, and J .N Ogbodo, Investigation of Egg Shell Waste as an Enhancer in the Carburization of Mild Steel, American Journal of Materials Science and Engineering, 2013,Vol.1, No. 2, 29- 33.

[7] P. Saigeeta, S. Tripurari, and A Gaurav, Effect of Post Carburizing Treatment on Hardness of Low Carbon Steel. International Journal of Advanced Mechanical Engineering, 2014, Volume 4, Number 7, pp. 763-766.

[8] R. A Higgins, Engineering Metallurgy, Viva Books Private Limited, New Delhi, 1998. 